ARTICLE

\title{
Development of Coupled Modeling System for Regional Water Cycle and Material Transport in the Atmospheric, Terrestrial, and Oceanic Environment
}

\author{
Haruyasu NAGAI ${ }^{*}$, Takuya KOBAYASHI, Katsunori TSUDUKI and Hiroaki TERADA \\ Japan Atomic Energy Agency, 2-4 Shirakata-shirane, Tokai-mura, Naka-gun, Ibaraki-ken, 319-1195, Japan
}

\begin{abstract}
Numerical simulations are the effective approach to investigate the complex mechanism in environmental phenomena and many numerical models have been developed for specific applications over wide range of field. Recently, much effort has been made to develop numerical models that can deal with the multi-physics problems in the complex environmental system. Most of these models are constructed by coupling existing models developed separately for their specific field of studies and called as coupled models.

A general-purpose model coupling program of Japan Atomic Energy Agency (JAEA-Coupler) has been developed to construct local to regional scale coupled models for environmental studies. In these coupled models, calculations of component models are carried out by different processors of parallel computers in the same manner as their original stand-alone calculations and the coupler controls these processes and handles data exchanges among component models using Message Passing Interface (MPI). Data exchanges are only conducted between the coupler and each component model and the coupler handles the data distribution to target models with interfacing spatial, temporal, and physical discrepancies among component models.

By using JAEA-Coupler, integrated coupled models to simulate regional water cycle and material transport have been constructed. The coupled model for regional water cycle consists of the non-hydrostatic atmospheric dynamic model of PSU/NCAR (MM5), detailed multi-layer land surface model (SOLVEG) and three-dimensional hydrology model (RIVERS) developed by JAEA, the third-generation ocean wind-wave model of NOAA (WW3), and the Princeton Ocean Model (POM). For the material transport, Lagrangian particle dispersion models for the atmosphere and ocean (GEARN and SEA-GEARN, respectively) developed by JAEA are incorporated into the water cycle coupled model, where RIVERS includes the function to calculate material transport. Test calculations were carried out to simulate water movement after heavy rainfall and dissolved material transport after a hypothetical release to the atmosphere. These results show the validity of the coupled models in appropriate data exchanges for complex interactions among component models and reasonable reproduction of the target phenomena.
\end{abstract}

KEYWORDS: model coupling, regional water and material transport, environmental studies, multi-physics problem, MPI

\section{Introduction}

Numerical simulations are the effective approach to investigate the complex mechanism in environmental phenomena and many numerical models have been developed for specific applications over wide range of research field. Recently, much effort has been made to develop numerical models that can deal with the multi-physics problems in the complex environmental system such as interactions among the atmosphere, land, ocean, and ecosystem. For example, coupled climate models have been developed and applied for climate researches. ${ }^{1-6)}$ Most of these models are constructed by coupling existing models developed separately for their specific field of studies and called as coupled models. Since the coupling of models developed separately with different architectures is not easy task, many software tools to support model coupling are proposed. As the coupling tools, the OASIS, Earth System Modeling Framework (ESMF), and Model Coupling Toolkit (MCT) are widely used to build many coupled models. ${ }^{7-9)}$ These

*Corresponding author, E-mail:nagai.haruyasu@jaea.go.jp software tools support multi-model coupling with controlling component model runs on parallel computers and exchanging data for interactions among component models, for example the Community Climate System Model (CCSM) consists of the atmosphere, land-surface, ocean, and sea-ice models coupled by the parallel coupler (Cpl6) developed using MCT. ${ }^{10,11)}$

A general-purpose model coupling program of Japan Atomic Energy Agency (JAEA-Coupler) has been developed to construct various coupled models for environmental studies. ${ }^{12)}$ The coupled model using JAEA-Coupler consists of a central coupler executable and separate component model executables. Calculations of component models are carried out by different processors of parallel computers in the same manner as their original stand-alone calculations and the coupler controls these processes and handles data exchanges among component models using Message Passing Interface (MPI). Data exchanges are only conducted between the coupler and each component model and the coupler handles the data distribution to target models with interfacing spatial, temporal, and physical discrepancies among component models. This coupling feature is similar to the Cpl6 for the 
CCSM, and very convenient for the construction of complicated coupled model dealing with multi-scale, multi-physics simulation such as water cycle and material transport in the atmospheric, terrestrial, and oceanic environments. The modifications of component models for coupling calculation are easy, simply adding some modules for data exchanges to each model code without changing the original structure of each model. It is also helpful to make a model code coupling with community models, which are updated regularly.

Although JAEA-Coupler essentially differs little from the Cpl6 and other coupling tools and does not encompass all functions incorporated into them (for example, efficient data exchange by parallel coupling in Cpl6, direct data exchange between component models, etc.), some advantages are distinguished in JAEA-Coupler depending on the target applications. While other coupling tools have been developed for climate modeling or earth system modeling for entire globe, JAEA-Coupler is intending to develop local to regional scale coupled models for water cycle and material transport, which is applied for prediction or mechanism elucidation of pollutants behavior in the environment. ${ }^{13,14)}$ For example, JAEA-Coupler can handle features of local to regional scale environmental models such as nested domains and Lagrangian particle dispersion, and also achieve very frequent data exchanges for complex interactions among multiple component models with integral time step of a few second. Local to regional scale coupled models are also developed by other coupling tools, ${ }^{15)}$ but these coupling are still limited. Similar to other coupling tools, JAEA-Coupler is also highly portable to be installed into most of parallel computational platforms. In practical sense, it is available on any platform where the mesoscale meteorological models MM5 and WRF, which are the most essential models in the local to regional scale coupled models, can be executed. ${ }^{16,17)}$ It can be installed in the same way as these component models without special settings to computational environment.

By using JAEA-Coupler, integrated coupled modeling system has been constructed and this system is applied to simulate regional water cycle and material transport with the combination of atmospheric, terrestrial, and oceanic models. ${ }^{12-14)}$ The design concept of the coupled modeling system is that they can easily produce optimum coupled models depending on the intended use by combining appropriate component models from many kinds of existing models. The coupled model for regional water cycle consists of the non-hydrostatic atmospheric dynamic model MM5, detailed multi-layer land surface model (SOLVEG) and three-dimensional hydrology model (RIVERS) developed by JAEA, ${ }^{18-20)}$ the third-generation ocean wind-wave model of NOAA, WAVEWATCH III (WW3), ${ }^{21)}$ and the Princeton Ocean Model (POM). ${ }^{22)}$ Concerning the coupling for ocean calculation (MM5, WW3, and POM), performance of the coupled model has already been demonstrated in the simulation of storm surge caused by Hurricane Katrina in August $2005 .^{23)}$ For the material transport in the environment, Lagrangian particle dispersion models for the atmosphere and ocean (GEARN and SEA-GEARN, respectively) developed by JAEA are incorporated into the water cycle coupled mod- $\mathrm{el}^{24,25)}$ where RIVERS includes the function to calculate material transport and SOLVEG and WW3 are not used. To show the validity and effectiveness of the coupled modeling system, these coupled models were applied to simulate the local water movement along the path of atmosphere, land surface, soil, river, and ocean after heavy rainfall and dissolved material transport after a hypothetical release to the atmosphere. These results show the validity of the coupled models in appropriate data exchanges for complex interactions among component models and reasonable reproduction of the target phenomena. Numerical experiments show the effectiveness of the system.

\section{Model Coupler}

\section{Design and Functions}

In the design concept of JAEA-Coupler, the emphasis is on its easiness in coupling and handling component models, which are developed separately with different architectures suitable for their specific field of studies, and at the same time, its high functionality in dealing with complex interactions among them. For this reason, we adopted the structure of coupled model with separate component model executables connected with a central coupler executable via the Message Passing Interface (MPI) on parallel calculations, where calculations of component models are carried out concurrently by different processors in the same manner as their original stand-alone calculations and the coupler controls these processes and handles two-way data exchanges among component models. This feature also reduces the effort for model coding. The modifications of component models are easy, simply adding some coupler modules and inserting some subroutine call statements into each model code. The coupler starts at first, then invokes and controls calculation processes and MPI communicators of component models. Data exchanges are only conducted between the coupler and each component model and the coupler handles the data distribution to target models with interfacing spatial, temporal, and physical discrepancies among component models.

To realize the above features, JAEA-Coupler provides the following primary functions.

- Coupling with multiple parallel models: Any numbers of models can be coupled in principle, although eight models can be coupled without any changes in the default setting of model code. Coupling with distributed memory parallel model is also supported.

- Interface for spatial and temporal discrepancies: The coupler holds the information of 3-D structured grids of component models and interface these spatial discrepancies by using interpolation schemes incorporated by user. Temporal discrepancies in integral time steps among component models are also handled by linear time interpolation for the data time requested by each component model. Therefore, each component model can send and receive data with its own grid in arbitrary time interval, which is prescribed in data exchange configuration file. 
- Exchanges of 1-D, 2-D, and 3-D array staggered grid data and particle data: The coupler supports data exchanges in the forms of 1-D, 2-D, and 3-D arrays and particle type. The above spatial interface is valid for array data and multiple grids for each component model can be assigned to deal with staggered grid. Particle type data can be also exchanged, which hold the information of position and some values for randomly distributed particles. Data exchange of this type can be extended to cope with the coupling of unstructured grid models.

- Handling of nested domain calculation: The coupler is applicable to the nested domain calculation, which is the important function of regional models such as MM5 and WRF. Each component model can have up to five nested domains and data exchange for each domain can be independently controlled by data exchange configuration file.

- File output of exchange data for offline coupling and visualization: Offline coupling calculations are available by using the coupler for the case of one-way data exchange. At first only sender models are executed and the coupler receives data from sender models and outputs them into files at each exchange time step. Then receiver models are executed by receiving exchange data from the coupler, which are read from the output files by the coupler at each exchange time step. The coupler can also output exchange data to be used for visualization. For this case, data for visualization from component models are interpolated onto the grid of one target component model and all data from component models can be visualized concurrently with the common coordinate system.

- Scalability for grid computing: The coupling function can be enhanced to be used between different types of parallel computers by using a MPI library named Stampi developed by JAEA. ${ }^{26)}$ This coupling method has been tested between the atmospheric model MM5 on a vector-parallel computer Fujitsu-VPP5000 and the land-surface model SOLVEG on a scalar-parallel computer Fujitsu-Primepower. ${ }^{27)}$ By using this enhanced coupling function, multi model coupling on grid computing environment can be realized.

\section{Coupler Processes}

In the following sections main processes of JAEA-Coupler are described. Process flows of the coupler and component models are schematically shown in Fig. 1.

\section{(1) Initialization}

The initialization process includes the coupler initialization and component models' initializations. In the coupler initialization, coupling conditions are set up according to a model configuration file that provides information on component models and data exchange configuration files that provide information on send/receive data for component models. Then, the coupler invokes component models specified by the model configuration file and establishes parent-child communication with each component model. If a component model is coded as distributed memory parallel model, it can be executed by multiple processors in the same

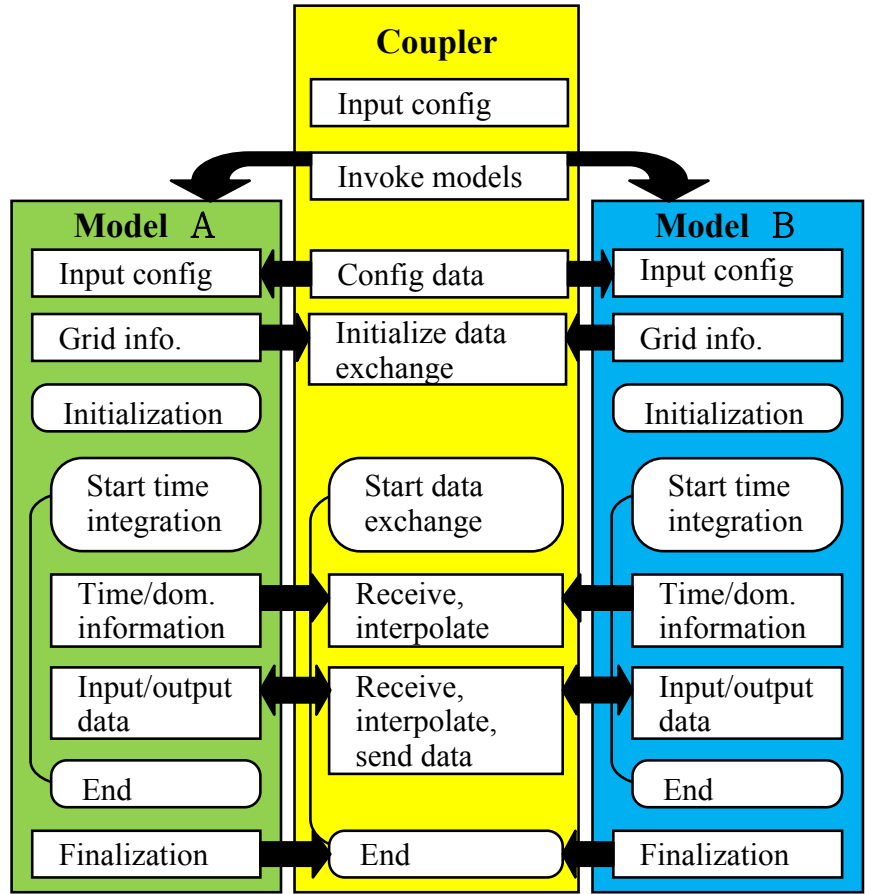

Fig. 1 Schematic illustration of the process flow in coupling calculation by JAEA-Coupler

way as the original standalone calculation of the model. In this case, the component model has its own local communication among multiple processes and the master process communicates with the coupler. After being invoked and given information on coupling conditions by the coupler, each component model sends data of grid and time settings to the coupler. The coupler gathers these data from all component models and establishes interfaces between all pairs of component models with data exchange.

\section{(2) Data Exchanges}

All data exchanges among component models are conducted through the coupler. Each component model can send and receive data at arbitrary points and number of times in each integration time step. Before sending and receiving data for new time in calculation or different domain for nested calculation, the information on these conditions is needed to be sent to the coupler. When the coupler receives a request to send data from a component model, it determines whether receiver models are running (concurrent coupling) or not (offline coupling) and stores received data together with the time and domain information on its memory area in case of concurrent coupling or in files in case of offline coupling. For a request to receive data from a component model, the coupler searches the requested data from its memory area or output files of the previous runs and sends the data to the component model if the data exist, where the data times are not always have to coincide with requested time and two data times around the requested one need to exist for interpolation. If the requested data are not to be found in output files or the memory area, the coupler aborts the coupling calculation with this error message in case of offline coupling or it makes the component model to be waiting for the 
data until the data come from the sender model in case of concurrent coupling. Therefore, the timing of receive data is carefully designed to be requested after the coupler received these data from sender models in case of multiple model coupling. Data stored on the memory area of coupler are eliminated after they have become unnecessary to be used for any temporal interpolations to make data requested by component models.

\section{(3) Spatial Interface}

The spatial interface among different grids of component models is important task for the model coupler that couples various kinds of existing environmental models, which are developed separately with different architectures suitable for their specific field of studies and uses grid systems with different resolution, coordinate system, map projection, etc. JAEA-Coupler provides the interface for spatial discrepancies among 3-D structured grids of component models. As mentioned in the initialization part, the coupler holds the information of grids for each component model and relationships between the grid points of sender and receiver models are established. For 2-D or 3-D grids, each grid point of receiver model is related to four or eight grid points of sender model that are located side-by-side including the grid point of receiver model within their rectangle box. The user needs to incorporate the interpolation scheme that defines how to determine the data at the point of receiver model by using data at the four or eight grid points of sender model, although a simple and common interpolation scheme for 2-D grids has been incorporated.

\section{Coupled Models}

\section{Coupled Model for Regional Water Cycle}

By using JAEA-Coupler, an integrated coupled model to simulate regional water cycle has been constructed. The coupled model consists of five models: the atmosphere (MM5), land-surface (SOLVEG), hydrology (RIVERS), ocean-wave (WW3), and ocean-current (POM) models as shown in Fig. 2. These component models and interactions among them are briefly described in the following sections.

\section{(1) Component Models}

The non-hydrostatic mesoscale atmospheric model MM5 is a community model having many users all over the world, and is used for many purposes, even for the official weather forecast by some countries. ${ }^{16)}$ It has many useful functions such as nesting calculations, 4-D data assimilation, and many options of parameterizations for cloud micro-physics, cumulus cloud, planetary boundary layer (PBL), radiation, and land surface scheme.

The land surface model SOLVEG consists of one-dimensional multi-layer sub-models for the surface atmosphere, soil, and vegetation, and a radiation scheme for the transmission of solar and long-wave radiation in the canopy. ${ }^{18,19)}$ It simulates diurnal variations and seasonal changes of variables in the surface atmosphere, soil, and vegetation canopy, and exchanges of energy and water among these systems, by using meteorological data of the surface layer atmosphere as the top boundary conditions. For coupling with an atmospheric model, one-dimensional model variables are expanded to three-dimensional ones whose horizontal coordinates coincide with those of atmospheric model. However, no interactions in horizontal directions are considered in the model.

The hydrology model RIVERS is a grid type distributed runoff model. ${ }^{20)}$ It consists of three-dimensional grid cells with five vertical layers and river channels. Horizontal and vertical water flows among connected grid cells and river channels are calculated from the GIS data (elevation, land-use, soil-type, etc.), and the water content of each grid cell is determined to keep the mass balance.

The ocean-wave model WW3 is known as third-generation wave model that is phase-averaged and stochastic. $^{21)}$ It simulates temporal and spatial variations of

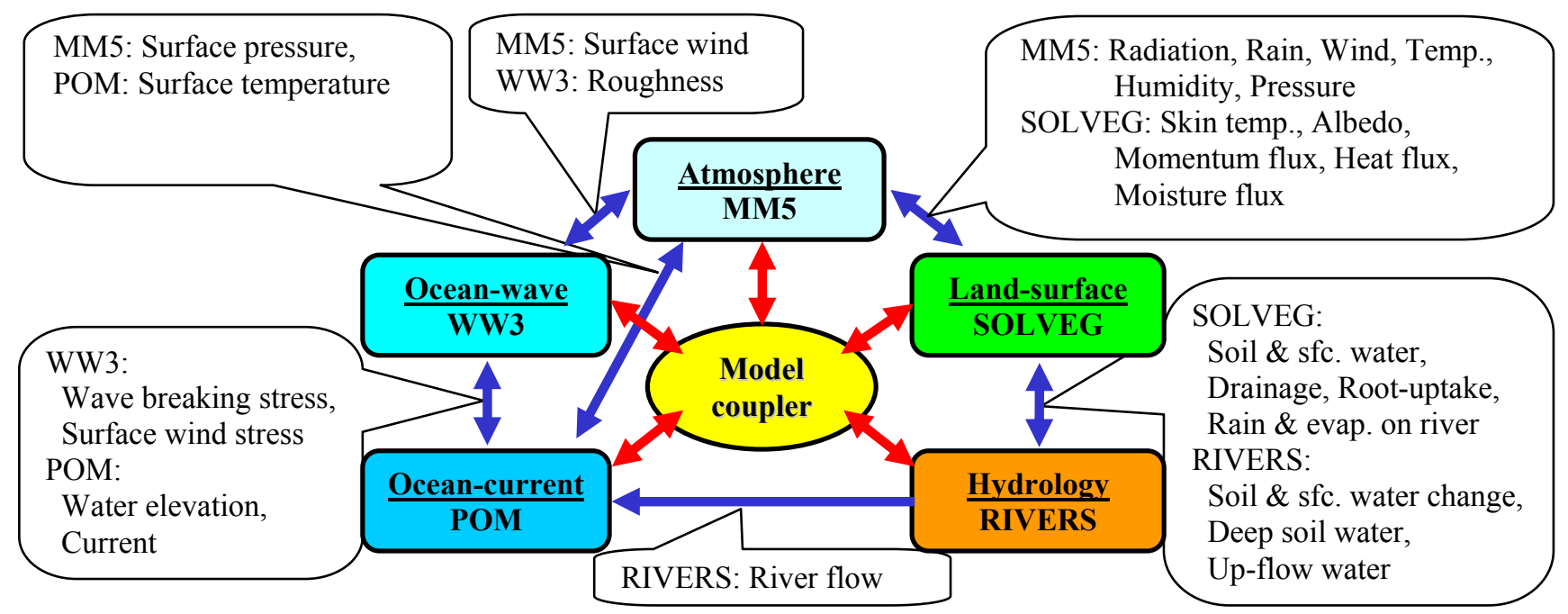

Fig. 2 Data exchanges and interactions among component models. Red arrows represent data exchanges via the JAEA-Coupler, and blue arrows are interactions among models, respectively. 
wave growth and decay resulting from the surface wind force, dissipation due to white-capping, and the bottom friction on the water column.

The Princeton Ocean Model POM is a three-dimensional primitive equation model, incorporating a turbulence closure model to provide a parameterization of the vertical mixing processes. ${ }^{22,28)}$ It calculates the three components of current velocity, salinity, temperature, turbulence kinetic energy, turbulence length scale, and free surface elevation as prognostic variables. It uses a terrain-following sigma coordinate and suitable for the coastal region.

\section{(2) Interactions among Component Models}

As shown in Fig. 2, interactions are divided largely into two systems; the atmosphere-ocean and atmosphere-land systems. For both systems, interactions occur through the sea or land surface, and WW3 or SOLVEG play the interface between the atmosphere and ocean or land.

For the atmosphere-ocean system, the momentum exchanges in the wind-wave-current system are considered. ${ }^{23)}$ The surface wind field from MM5 provides the wind stresses; the wave-induced stress and turbulence-induced stress to generate wind-wave in WW3, and the surface stress to generate current in POM. For the wave calculation in WW3, the surface current and water elevation, which are provided from POM, are necessary as well as the wind stress. Calculated wave breaking stress and surface roughness length are offered to POM and MM5, respectively. POM calculates the surface current using the wind stress from MM5 and wave breaking stress from WW3. It also simulates surface water elevation using sea surface pressure from MM5, and this output together with the surface current are provided to WW3. Although the energy and water exchanges are also considered in the atmosphere-ocean interaction, only the sea surface temperature by POM is provided to MM5 as the surface boundary condition.

For the atmosphere-land system, the momentum, energy, and water exchanges between the atmosphere and land-surface are considered completely. MM5 sends the surface layer variables: radiation (short and long-wave), precipitation, wind speed, temperature, humidity, and air pressure, to SOLVEG at every time steps. With these inputs, SOLVEG calculation proceeds for the same time interval as MM5 and sends its results to MM5: skin temperature, albedo, momentum flux, heat flux, and vapor flux. MM5 receives these values in the next time step and uses them as the surface boundary condition in the PBL process instead of those by the land surface model included in MM5. For the interaction between the land-surface and hydrology, only the water exchange is treated. SOLVEG provides detailed calculations of surface water and near-surface soil water contents, and vertical fluxes of soil water to RIVERS. With these inputs, RIVERS calculates the river flow, deep layer soil water content, and three dimensional movement of soil water, and the changes due to horizontal water movements are fed back to SOLVEG. These exchanges enable SOLVEG to consider the horizontal movement of soil water, which is not simulated in vertical one-dimensional calculations.

\section{Coupled Model for Material Transport}

By incorporating material transport models into the coupled water cycle model described above, an integrated coupled model to simulate material transport in the multiple (atmospheric, terrestrial, and oceanic) environments has been constructed. The coupled model consists of five models: the atmosphere (MM5), atmospheric dispersion (GEARN), hydrology-transport (RIVERS), ocean-current (POM), and oceanic dispersion (SEA- GEARN) models as shown in Fig. 3. The land surface model SOLVEG and ocean wave model WW3 have not been used in this coupling system, because material transport models for these parts have not been developed. Component models for material transport and interactions among them are briefly described in the following sections.

\section{(1) Component Models}

Lagrangian particle dispersion model GEARN calculates the atmospheric dispersion of radionuclides by tracing the trajectories of a large number of marker particles discharged from a release point. ${ }^{24)}$ By using meteorological field predicted by MM5, it calculates the movement of each particle affected by both advection due to mean wind and subgrid scale turbulent eddy diffusion. The combination of MM5 and GEARN provides the capability of atmospheric dispersion prediction for the Japanese emergency response system WSPEEDI (Worldwide version of System for Prediction of Environmental Emergency Dose Information). ${ }^{29)}$ GEARN also has a function of nesting calculation for two domains corresponding to MM5's nested domains. As shown in Fig. 3, two nested domains of GEARN are calculated separately by different executables, and marker particles that flow out and in across the boundary of inner domain are exchanged between domains.

The oceanic dispersion model SEA-GEARN is a Lagrangian particle dispersion model similar to GEARN. ${ }^{25)}$ By using oceanic field predicted by POM, it calculates the movement of each particle caused by the transport due to ocean current and the diffusion due to turbulence.

Unlike the atmospheric and oceanic dispersion, the function to calculate the material transport in soil and river is included in RIVERS. It also uses Lagrangian particle dispersion model. However, this function has not been completed and is applicable only for a dissolved matter.

\section{(2) Interactions among Component Models}

Interactions considered in this coupled model are shown in Fig. 3. The atmospheric and oceanic dispersion models GEARN and SEA-GEARN receive the three dimensional meteorological and oceanic field predicted by MM5 and POM, respectively, while RIVERS uses the precipitation and evapotranspiration calculated by MM5 as the surface boundary condition for water movement. For material transport, two-way exchange between the atmosphere and land/sea surface, and one-way exchange between land and ocean are considered. Airborne materials move to the land or sea surface through dry and wet deposition. Volatile materials in land and ocean can be emitted to the atmosphere. Materials 


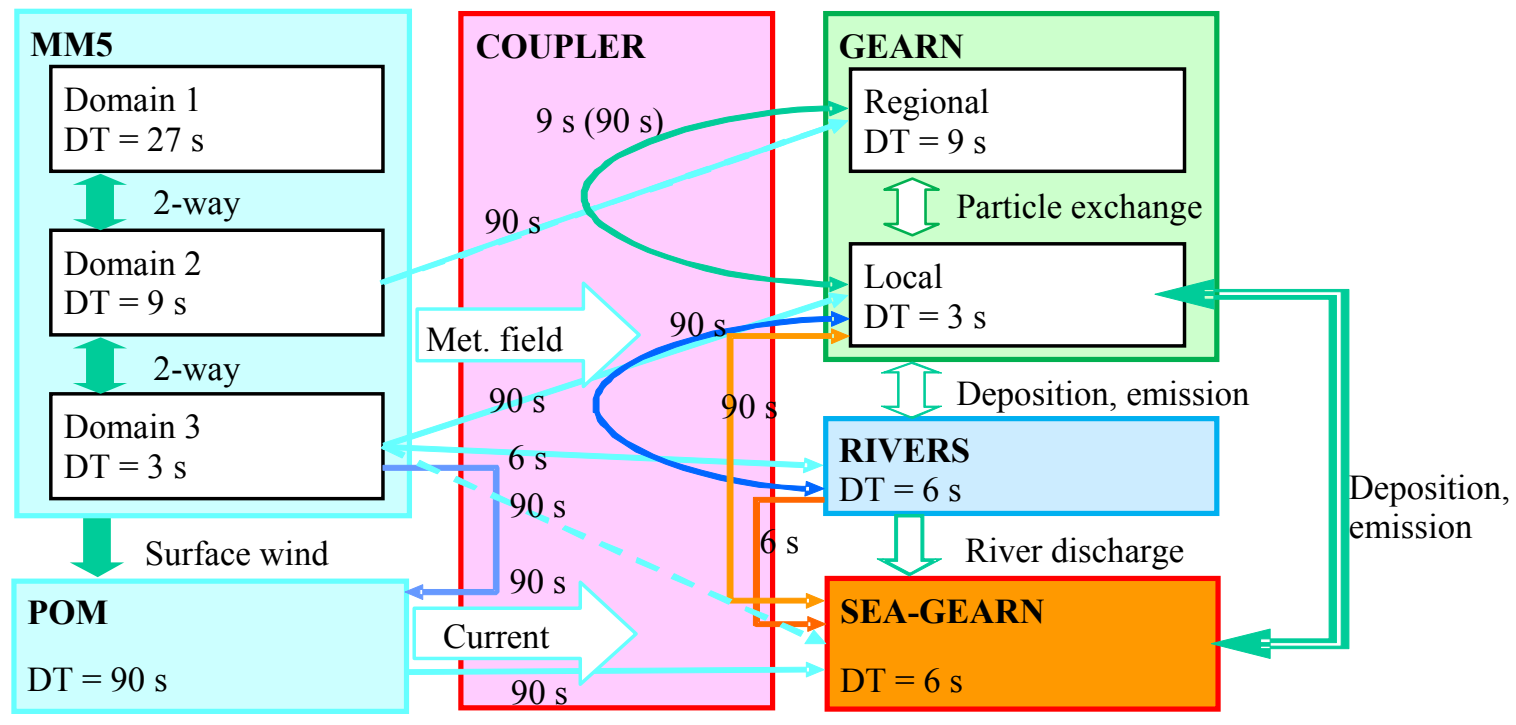

Fig. 3 Data exchanges among component models for material transport coupling

dissolved in river water are discharged to the ocean from estuaries. These interactions among GEARN, RIVERS, and SEA-GEARN are handled by JAEA-Coupler via data exchange.

\section{Test Simulations}

Test simulations were carried out to examine the performance of coupled models. Target area is about $130 \mathrm{~km}$ square area of Eastern part of Japan including two major river basins, between which JAEA is located, as shown in Fig. 4. The event of flow increase at these rivers after a heavy rainfall on 26 December 2006 was chosen for test calculations. Three-domain nesting is used for MM5 and the other models are coupled with the most inner domain (Fig. 4). Calculation periods are five and seven days from 00UTC on 24 December 2006 for the water cycle and material transport calculations, respectively.

\section{Regional Water Cycle Calculation}

Meteorological forecast dataset for Japan area (GPV-RSM) provided by Japan Meteorological Agency (JMA) was used for MM5 input and RIVERS spin-up calculation (more than three months from 1 September 2006) for soil water and river flow. The grid resolution of GPV-RSM data is horizontally $20 \mathrm{~km} \times 20 \mathrm{~km}$ and vertically 17 pressure levels. Time interval is three hours.

Three nested domains D1, D2, and D3 were used for MM5 calculation. Horizontal grid points were $100 \times 100$ for D1 and D2 and $130 \times 130$ for D3, and vertical 23 layers were used for all the domains. Grid intervals for D1, D2, and D3 were 9, 3, and $1 \mathrm{~km}$, respectively. Time increments for D1, D2, and D3 were 27, 9, and 3 seconds, respectively. Physics options were set as follows. No cumulus parameterization, microphysics with Schultz scheme, cloud radiation, MRF-PBL scheme, and Noah-LSM were used. SOLVEG used horizontal $130 \times 130$ grid with $1 \mathrm{~km}$ resolution (same as MM5-D3) and vertically 10 layers for canopy air (lower (a) MM5

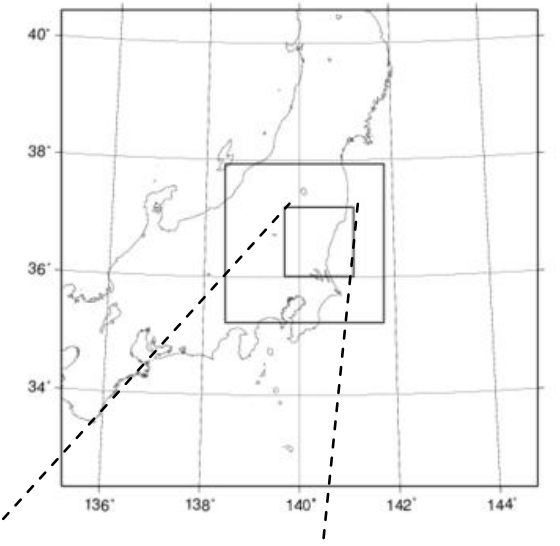

(b) SOLVEG, RIVERS'

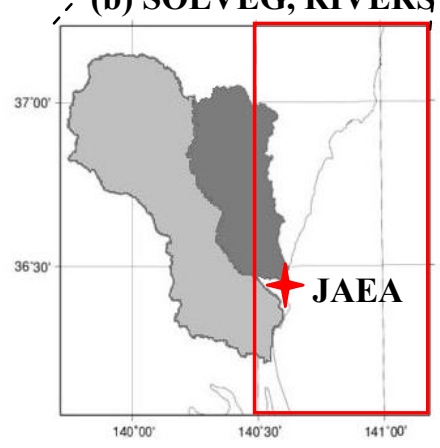

(c) WW3, POM

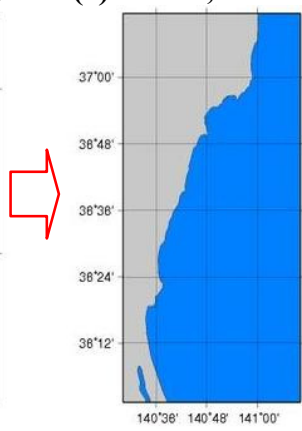

Fig. 4 Calculation domains for test calculations of coupled models: (a) MM5 three nested domains, (b) SOLVEG and RIVERS domains, (c) WW3 and POM domains. Shaded areas in (b) represent two major river basins around JAEA shown by a red cross.

five layers) and atmosphere up to $10 \mathrm{~m}$ above the canopy and six soil layers down to $1 \mathrm{~m}$ below the land surface. Time increment was 6 seconds. RIVERS used horizontal $130 \times 130$ grid with $1 \mathrm{~km}$ resolution (same as MM5-D3 and SOLVEG) and vertical layers with one surface, four soil layers, and one river. Time increment was 6 seconds. POM 
used horizontal $26 \times 54$ grid with $3 \mathrm{~km}$ resolution and vertically 21 layers. Time increment was 60 seconds for internal mode and 2 second for external mode. WW3 used horizontal $26 \times 54$ grid with $3 \mathrm{~km}$ resolution (same as POM). Time increment was 60 seconds.

A scalar-parallel computer SGI-Altix $3700 \mathrm{Bx} 2$ of JAEA was used to execute coupled calculations. Parallel calculations were used for MM5 (12 CPUs), SOLVEG (10 CPUs), and WW3 (6 CPUs), while RIVERS and POM calculations were single. JAEA-Coupler uses 2 CPU (1 CPU for MPI process). Therefore, total number of used CPU was 32. The calculation for five day period by uncoupled MM5 and those of two different combinations of models, MM5-SOLVEG-RIVERS and MM5-RIVERS, were also carried out to examine the influences of the coupling condition on computational costs.

\section{Material Transport Calculation}

In the material transport calculation, the same configurations were used for MM5 and RIVERS as those for the water cycle calculation. POM used the same domain as the water cycle calculation but the time increment was 90 seconds for internal mode and 3 second for external mode in this case. For the atmospheric dispersion model GEARN, the same domain and time increment as MM5-D3 were used. In order

(a) MM5 original

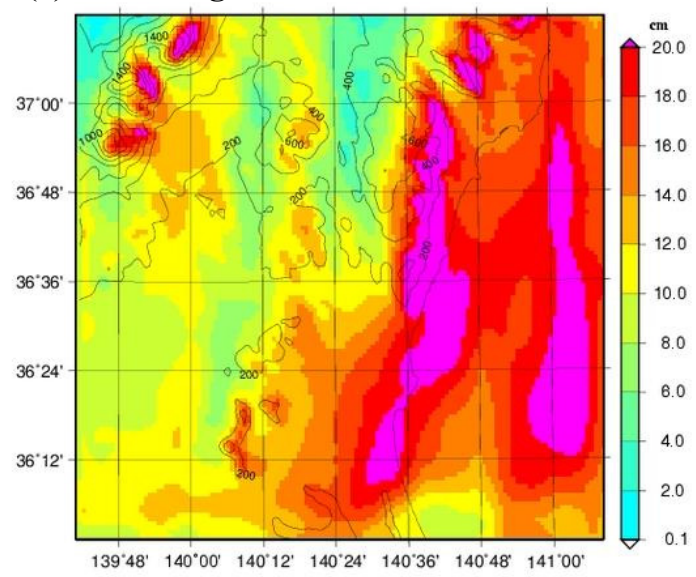

(b) Coupled model

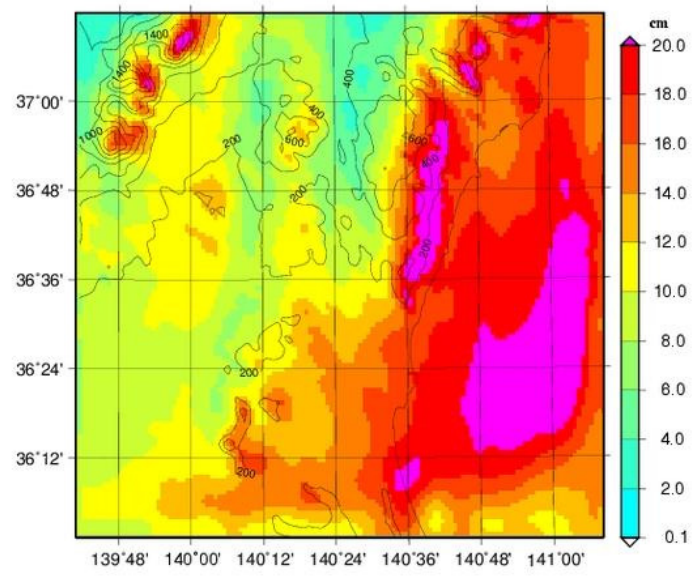

Fig. 5 Calculated daily rainfall patterns on 26 December 2006 by (a) the original MM5 and (b) coupled model to reduce computational costs the function of nesting calculation as shown in Fig. 3 was not applied in this test calculation. The oceanic dispersion model SEA-GEARN used the same domain as POM and the time increment was 6 second. Time steps of data exchange via the model coupler were set as shown in Fig. 3.

Hypothetical release condition was used to examine the material transport circulation in the multiple environments. Atmospheric discharge of tritiated water vapor (HTO) was assumed as a completely dissolved and volatile matter that has two-way exchange between the atmosphere and land/sea surface. The release point was located at the JAEA site $(36.45 \mathrm{~N}, 140.60 \mathrm{E}$; Fig. 4$)$ and $50 \mathrm{~m}$ above the ground. The release period with constant release rate $\left(10^{13} \mathrm{~Bq} / \mathrm{h}\right)$ was three hours from 12UTC on 26 December 2006. Moderate wind blew from the Pacific Ocean toward inland direction and heavy rainfall event started around this period. Therefore, the released HTO is expected to move toward inland and mostly deposit on the land surface through wet deposition. In test calculation, the effect of rainfall on the HTO migration in the multiple environments was examined by numerical experiment, reducing rainfall amount used in RIVERS and GEARN calculations, which was provided by MM5 through data exchange via JAEA-Coupler.

A PC-cluster was used for this calculation. MM5 used 2 CPUs and $1 \mathrm{CPU}$ was assigned to each of other models: RIVERS, POM, GEARN, and SEA-GEARN. With 2 CPUs for JAEA-Coupler, this coupling calculation used 8 CPUs.

\section{Results}

\section{Regional Water Cycle Calculation}

For five-day calculation of this test case, it took about 27 hours by the five-model coupling using 32 CPUs. For the other combinations of models, it took about 22, 24 and 27 hours for the same case by the uncoupled MM5 using 12 CPUs, MM5-RIVERS coupling using 19 CPUs, and MM5-SOLVEG-RIVERS coupling using 25 CPUs, respectively. Frequent data exchanges between MM5 and SOLVEG (every 6 seconds) and those between SOLVEG and RIVERS (every 6 seconds) are the reason for the increase of computation time. Concerning the ocean models, data exchanges are carried out in 900 seconds interval and times spent for these procedures are negligible.

Calculated daily rainfall patterns on 26 December 2006 by the original MM5 and coupled model are shown in Fig. 5. As comparing with rainfall patterns by other combinations of coupling, the rain pattern predicted by MM5 was changed mostly by feeding back the land surface boundary conditions by SOLVEG, although effects of feedback by other models were detected apparently. Time series of hourly rainfalls and river flow rates are compared between calculations by the coupled model and observations at the points within one of the main river basins (Naka River) shown in Fig. 6. Figures 7 and 8 show rainfall and river flow rate comparisons, respectively. The heavy rainfall event was occurred on 26 December, and the coupled model reproduced both temporal change and rain intensity successfully at most of the points. The model results have a tendency of under and overestima- 
tion during early and late period of rainfall event, respectively. This tendency is more significant at eastern part, especially at the point near estuary (Fig. 7(e)). It is considered that this tendency in calculated rainfall caused the slight delay in initial increase of river flow and overestimation of river flow rate at lower reach of the river as shown in Fig. 8. Although rainfall calculation needs the improvement with further investigation, the coupled calculations from rainfall to river discharge through the land surface process are reasonable considering the relationship between the rainfall pattern and river flow rate. Since the mass conservation of water among the component models was confirmed throughout the calculation period by examining output of each model, the coupled calculation and data exchange via JAEA-Coupler was also validated in this test calculation.

\section{Material Transport Calculation}

For 4.5 days calculation after the release start time, it took about 50 hours by the five-model coupling using 8 CPUs. In numerical experiments, rainfall amount was reduced from the coupled calculation (control case) to one tenth (0.1-rain case), one hundredth (0.01-rain case), and zero (no-rain case). Computational time changed from 50 hours for the control case to 68, 66, and 54 hours for 0.1-rain, 0.01-rain, and no-rain cases, respectively. The difference in computational times in these cases was caused by change in computational load of SEA-GEARN due to the particle number difference depending on the input of HTO amount from the atmosphere and river. Since the total amount of HTO, i.e., the sum of all portions distributed to the component models was conserved throughout the calculation period as shown in figures below, the coupled calculation and data exchange for the material transport was also validated in these test calculations.

The difference of material transport calculated by the coupled model from the original GEARN is shown by comparing the deposition pattern change for the 12 hours after

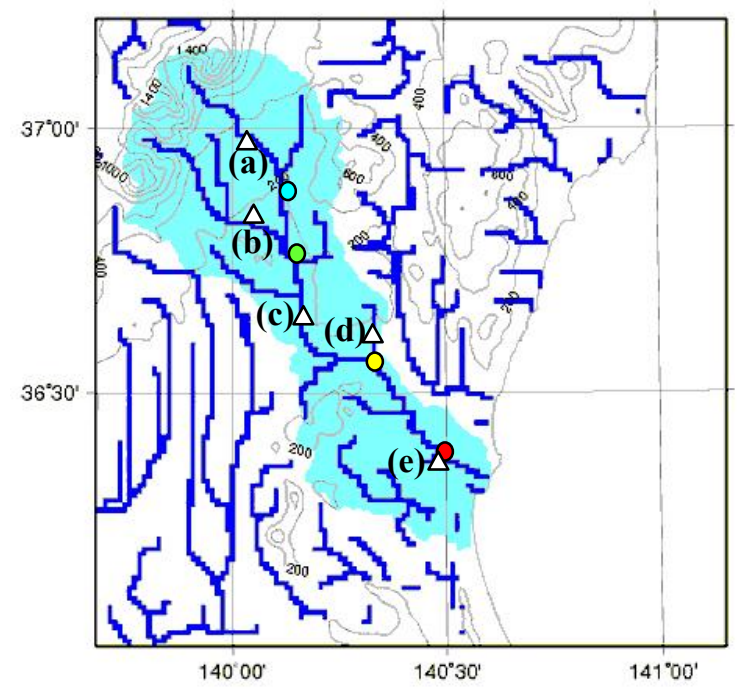

Fig. 6 Observation points for rainfall and river flow rate in Naka River basin. Triangles and circles represent rainfall and river flow rate observation points, respectively. (a)

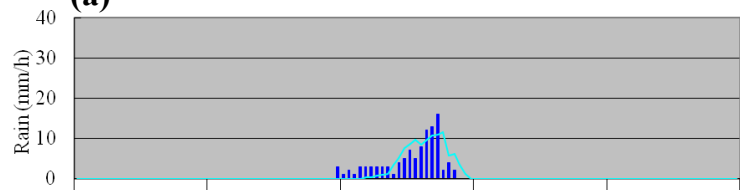

(b)

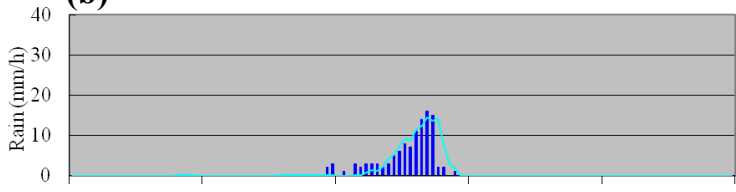

(c)

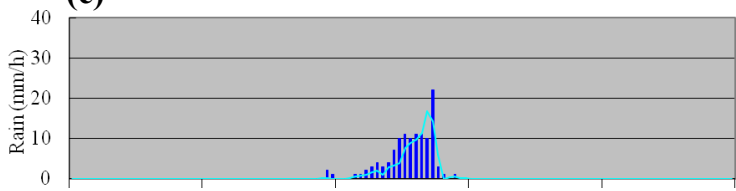

(d)

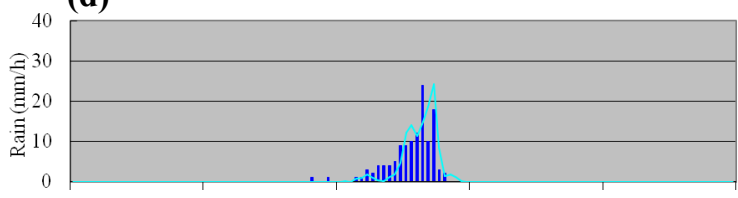

(e)

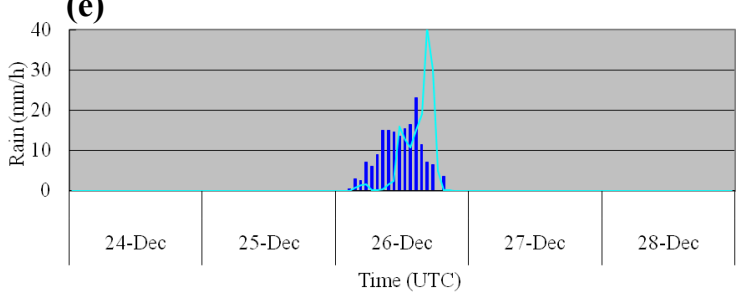

Fig. 7 Time series of hourly rainfalls by observation (bars) and calculation (lines) by the coupled model at the points (a) to (e) shown in Fig. 6

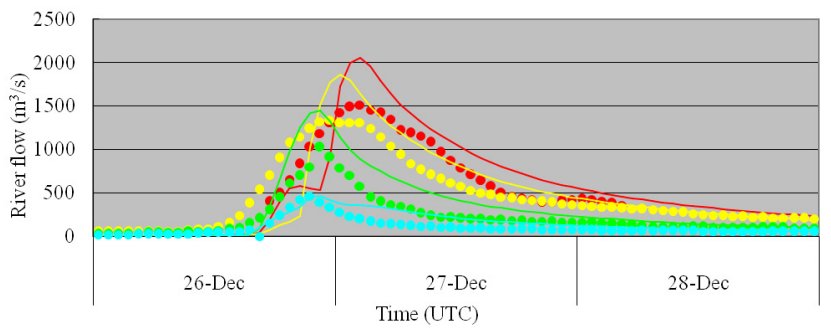

Fig. 8 Time series of river flow rates by observation (circles) and calculation (lines) at the points of circles with corresponding color shown in Fig. 6

HTO release time in Fig. 9. In the original GEARN calculation, most of the released HTO flew out the calculation domain 3 hours after the release end ( 6 hours after the release start time) and the deposition pattern had little change after that time. While the deposition pattern by the coupled model is almost the same as the original GEARN at the release end time, it changes significantly from the original one after that time. Two major processes newly added in the coupled model, which are not considered in the original GEARN, caused this difference. One is runoff of deposited 
(a) Original GEARN
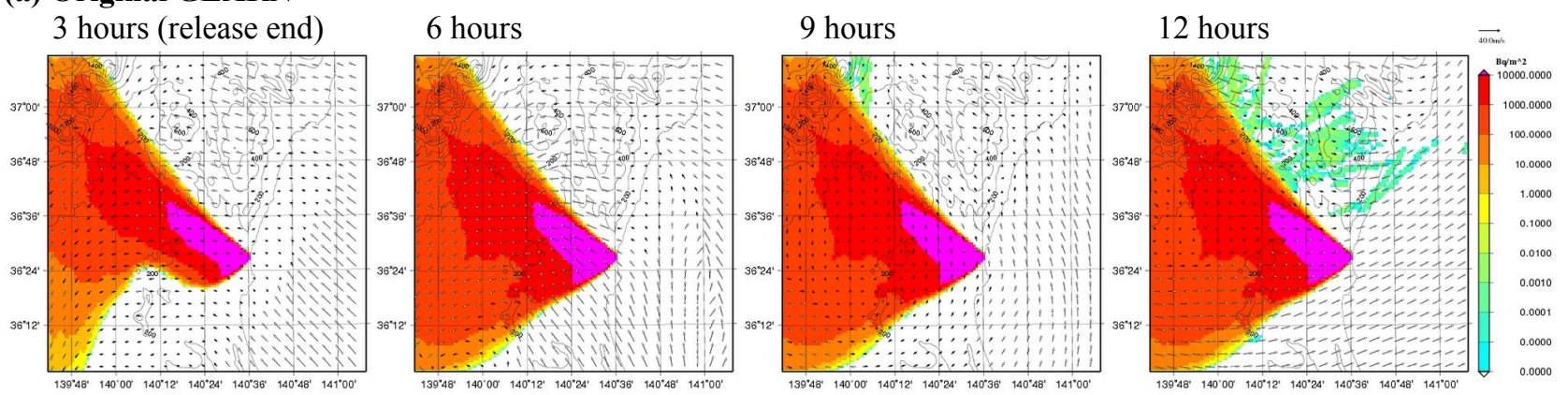

(b) Coupled model
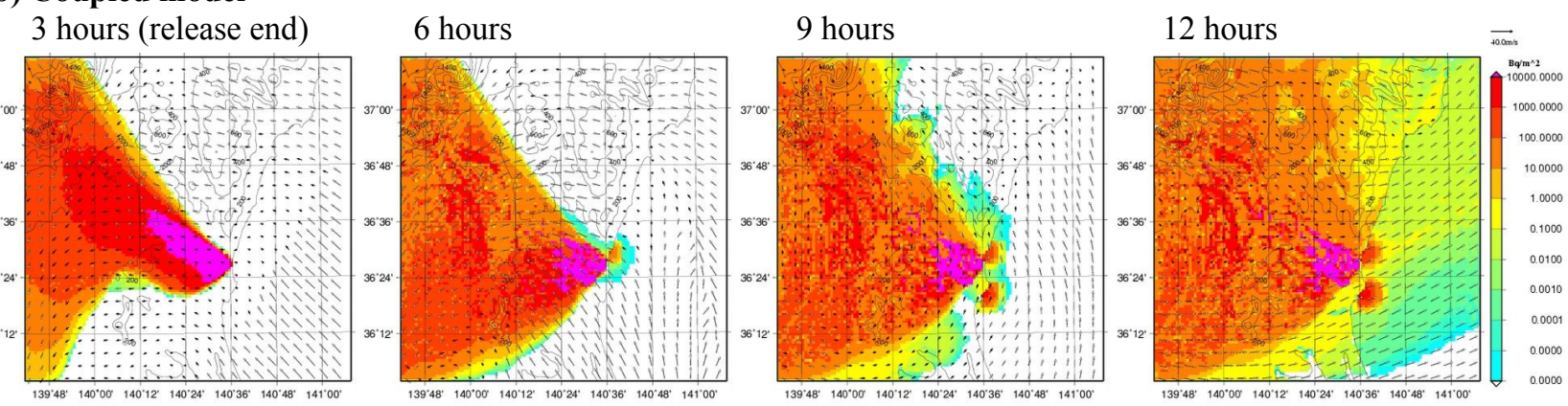

Fig. 9 HTO deposition pattern change by (a) the original GEARN and (b) coupled model for 12 hours after release start

HTO to rivers calculated in RIVERS. The other is re-emission of HTO from the land and ocean surfaces. Due to the former process, HTO deposited on the land surface flew out to rivers after the heavy rainfall, and the deposition pattern was changed completely, and the deposited HTO level decreased drastically. HTO in rivers was discharged into the ocean and the contaminated area was extended. The latter process also caused rapid decrease of deposited HTO level, and secondary plumes due to the re-emission resulted into the extension of contaminated area, too. This result indicates that the migration of dissolved and volatile matter like HTO in the multiple, or actual, environments cannot be predicted properly by conventional dispersion models like the original GEARN and this kind of coupled model is necessary.

In the numerical experiment, the effect of rainfall on the migration of HTO in the multiple environments was investigated. Figure 10 shows HTO deposition patterns at 12 hours after release start time by the coupled model for control case (heavy rainfall), 0.1-rain case (ordinary rainfall), 0.01-rain case (light rainfall), and no-rain case. The result indicates that the deposition pattern is changed by the influence of rainfall intensity. The HTO deposition increases with the rainfall intensity due to the increase of wet deposition. However, as the complete dissolved matter, the runoff of deposited HTO to rivers also increases with the rainfall intensity, and the amount of deposited HTO decreases at the heavy rain condition (control case). Temporal changes of HTO amounts in the atmosphere, land, river, and ocean of calculation domain, and those flowed out the domain from atmosphere, land, and ocean calculated by the original GEARN and coupled model are shown in Figs. 11 to 13. As shown in Fig. 11, the original GEARN calculates only HTO amounts in the atmosphere, surface deposition, and outside (a) Control case

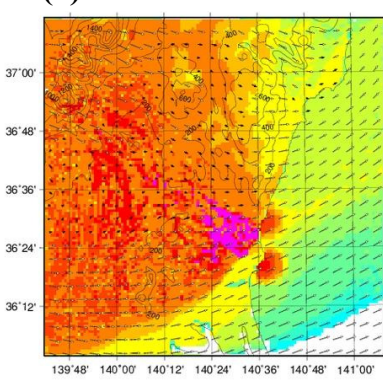

(c) 0.01-rain case

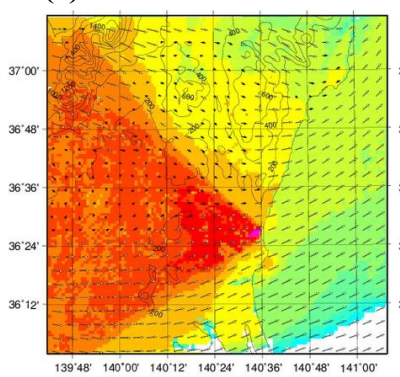

(b) 0.1-rain case

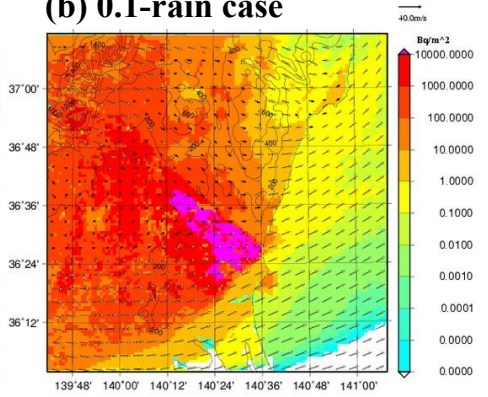

(d) No-rain case

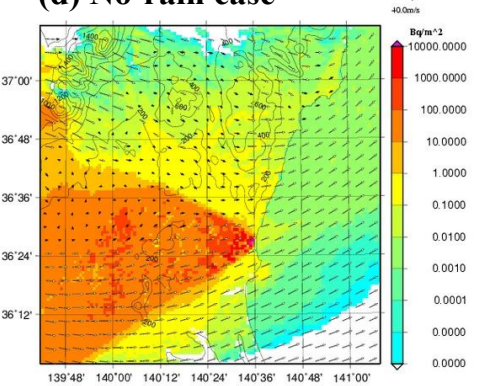

Fig. 10 HTO deposition pattern at 12 hours after release start by coupled model for (a) control case, (b) 0.1-rain case, (c) 0.01-rain case, and (d) no-rain case

of the domain. As mentioned above, HTO stays in the atmosphere only for the first 6 hours after the release start time, then, it is distributed into two parts; the deposition (about 95\%) and outflow (about 5\%). The deposited HTO decreases slightly only with the radioactive decay (half life: 12.33 years).

In the coupled calculation, temporal changes of HTO amounts in the atmosphere, land, and ocean differ consider- 
ably with the rainfall conditions. In the control case of coupled calculation (Figs. 12(a) and 13(a)), temporal changes of HTO amounts are similar to the original GEARN calculation during the first 6 hours, but completely different after that time. The deposition part decreases rapidly with the runoff to rivers and re-emission to the atmosphere. HTO also remains in the atmosphere throughout the calculation period due to re-emission from land and ocean surfaces, and the amount shows diurnal variation corresponding to the higher re-emission rate during daytime. The river discharge causes the increase of HTO amount in the ocean up to about $12 \%$ of the total HTO release. The outflow from the domain is much larger than the original GEARN calculation, and amounts of the atmospheric outflow and terrestrial outflow are about $17 \%$ and $19 \%$ of the total release, respectively. The re-emission of HTO from land and ocean surface increases the atmospheric outflow by more than three times. The HTO remaining on the land decreases to about $51 \%$ of the total release at the end of calculation.

By decreasing rainfall intensity, the distribution pattern of released HTO changes drastically as shown in Figs. 12 and 13. As mentioned above, the HTO deposition at 12 hours

(a)

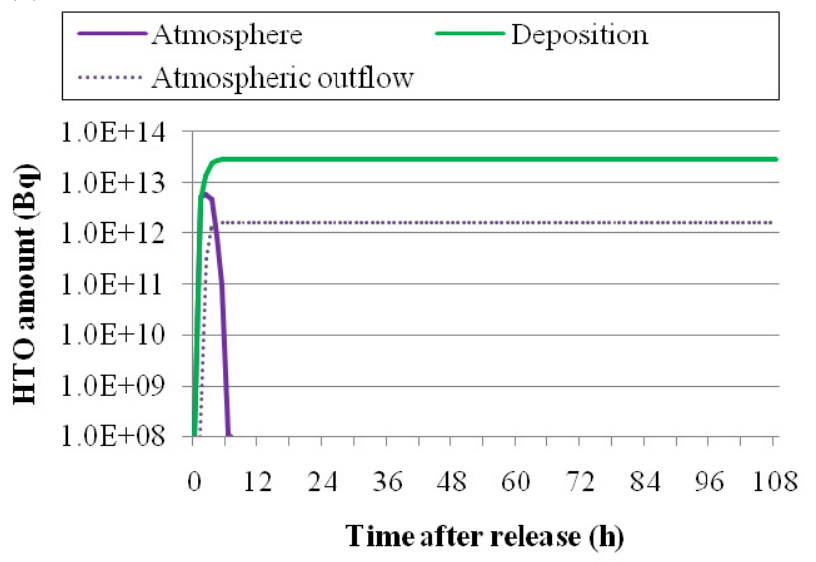

(b)

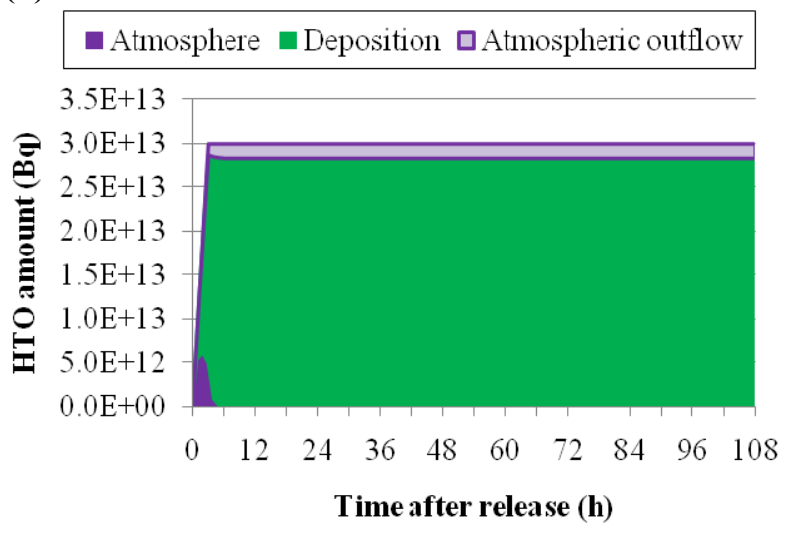

Fig. 11 Temporal changes of HTO amounts in the atmosphere and surface deposition in calculation domain, and HTO amount flow out the domain calculated by the original GEARN in (a) line chart and (b) area chart after the release start is the largest for the 0.1-rain case, and decrease with rainfall intensity (Fig. 10). This tendency is also shown in Figs. 12 and 13. However, the deposited HTO amount for the 0.1 -rain case decreases rapidly after that time, and becomes about $27 \%$ of the total release at the end of calculation. Meanwhile, the amount of HTO in the atmosphere for the same case increases around this period and has the maximum value (about $25 \%$ of the total release) at 13 hours after the release start time. The re-emission rate around this period for the 0.1-rain case is higher than that for the control case. It is because most part of the deposited HTO remains on the land surface and is easy to evaporate for the 0.1-rain case, while the HTO amount on the land surface is small for the control case due to the infiltration into the soil with the heavy rainfall. As the result of these processes, about $70 \%$ of the total release flows out the calculation domain from the atmosphere in the 0.1-rain case.

Concerning the 0.01-rain and no-rain cases, the HTO deposition decreases significantly and the HTO amount in the atmosphere becomes large for the first 6 hours with the peak value of about two thirds of the total release. Most HTO (97\% for 0.01 -rain case and $99 \%$ for no-rain case) of the total release flows out the calculation domain from the atmosphere for these cases.

\section{Conclusion}

A general-purpose model coupling program JAEA-Coupler has been developed and applied to construct integrated coupled models to simulate regional water cycle and material transport. To examine the performance of JAEA-Coupler and the coupled models, test calculations were carried out to simulate water movement after heavy rainfall and dissolved material transport after a hypothetical release to the atmosphere. These results show the effectiveness of JAEA-Coupler and the validity of coupled models in appropriate data exchanges for complex interactions among component models and reasonable reproduction of the target phenomena.

It was shown in the numerical experiments controlling rainfall intensity by the data exchange via JAEA-Coupler that the distribution of released HTO in the multiple environments changes significantly by the influence of water cycle change. The result by the coupled model is also considerably different from the original GEARN calculation. It is because that the original GEARN does not consider two major processes newly added in the coupled model, i.e., the runoff of deposited HTO to rivers and re-emission of HTO from the land and ocean surfaces. These results indicate that this kind of coupled model is necessary and effective to predict properly and investigate the migration of dissolved and volatile matter like HTO in the multiple environments.

\section{Acknowledgment}

Test calculations in this study used a scalar-parallel computer SGI-Altix3700Bx2 operated by the Center for Computational Science \& e-System of JAEA to execute the coupled water cycle model. 
(a) Control case
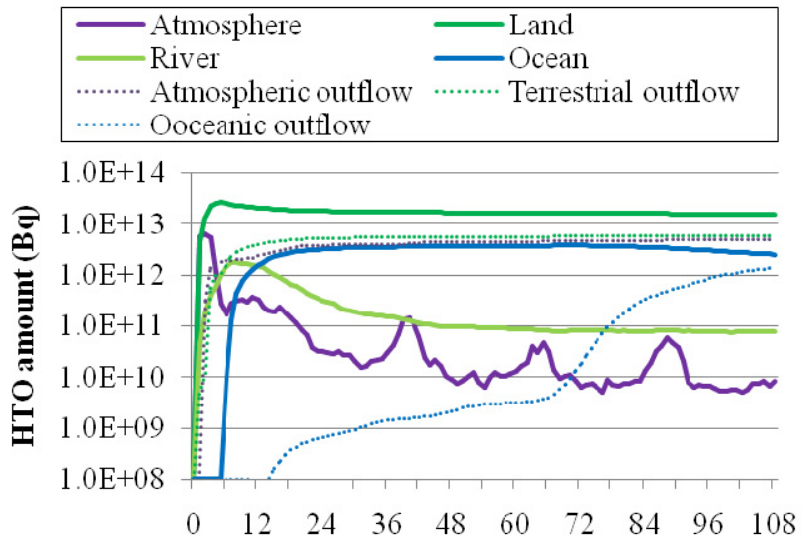

(b) 0.1-rain case

Time after release (h)

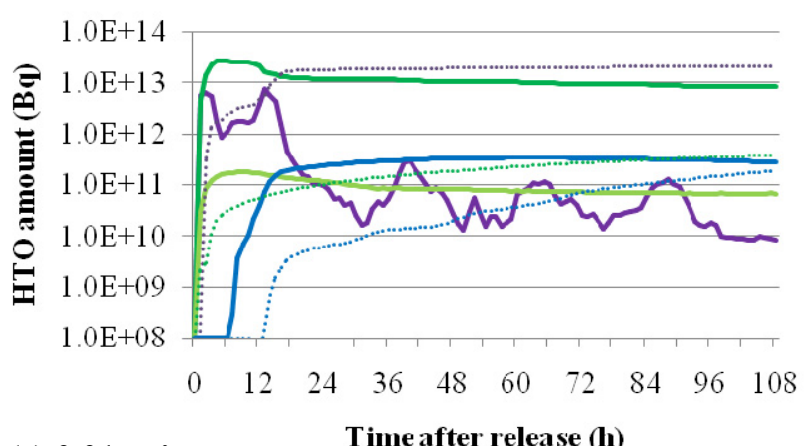

(c) 0.01-rain case

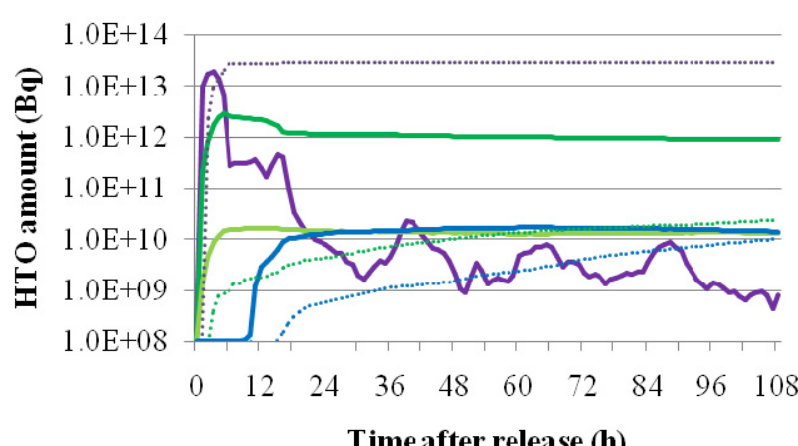

(d) No-rain case

Time after release $(h)$

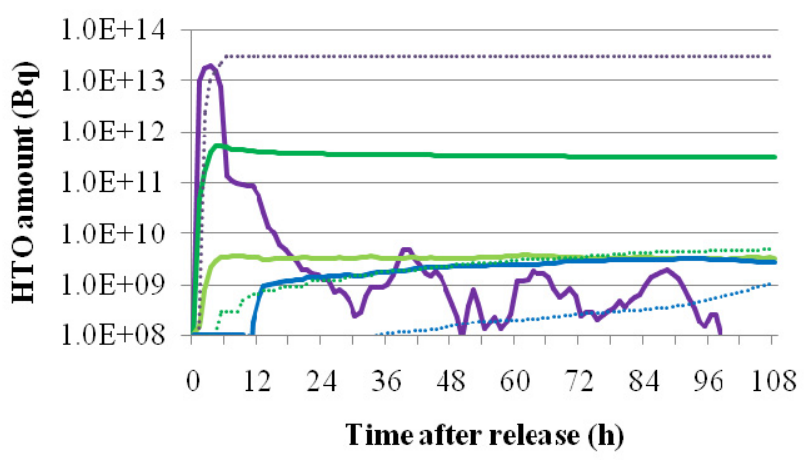

Fig. 12 Temporal changes of HTO amounts in the atmosphere, land, river, and ocean of calculation domain, and those flowed out the domain from atmosphere, land, and ocean calculated by coupled model for (a) control case, (b) 0.1-rain case, (c) 0.01-rain case, and (d) no-rain case (a) Control case
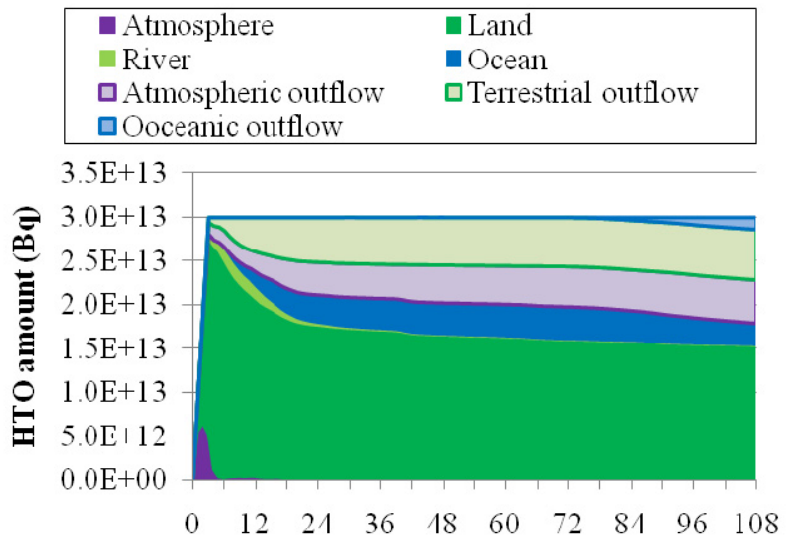

(b) 0.1-rain case

Time after release $(h)$
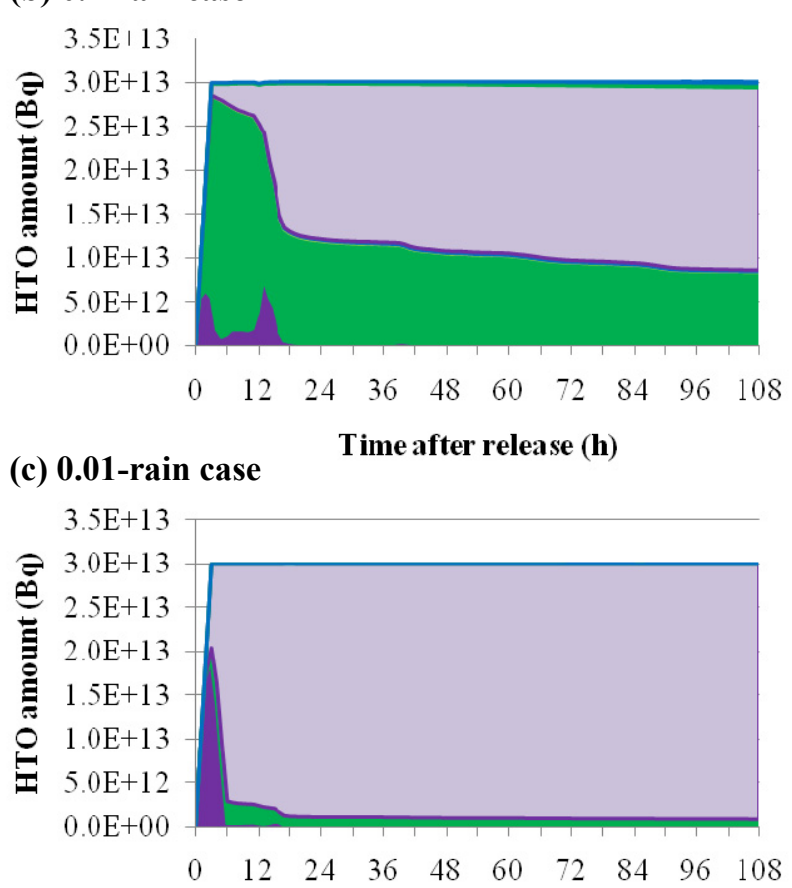

(d) No-rain case

Time after release $(h)$

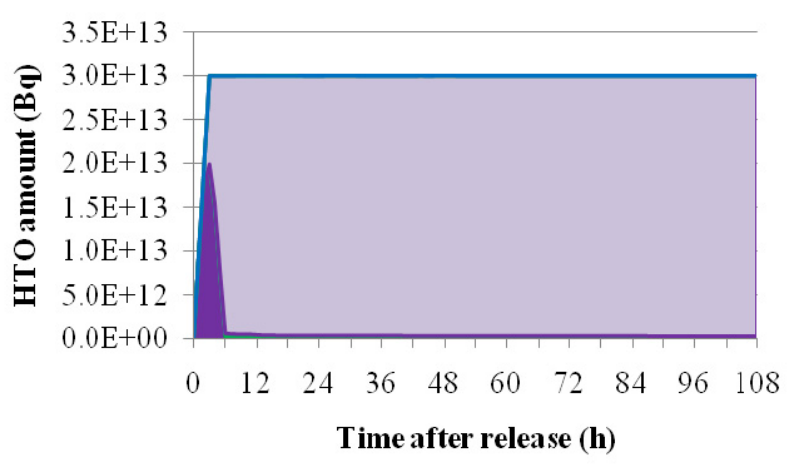

Fig. 13 Same as in Fig. 12 but in the form of area chart 


\section{References}

1) S. Manabe, R. J. Stouffer, "Low-frequency variability of surface air temperature in a 1000-year integration of a coupled atmosphere-ocean-land surface model," J. Climate, 9[2], 376-393 (1996).

2) B. A. Boville, P. R. Gent, "The NCAR Climate System Model, Version One," J. Climate, 11[6], 1115-1130 (1998).

3) G. L. Russell, D. Rind, "Response to $\mathrm{CO}_{2}$ transient increase in the GISS coupled model: Regional coolings in a warming climate," J. Climate, 12[2], 531-539 (1999).

4) D. G. Dewitt, E. K. Schneider, "The processes determining the annual cycle of equatorial sea surface temperature: A coupled general circulation model perspective," Mon. Wea. Rev., 127[3], 381-395 (1999).

5) S. Emori, T. Nozawa, A. Abe-Ouchi, A. Numaguti, M. Kimoto, T. Nakajima, "Coupled ocean-atmosphere model experiments of future climate change with an explicit representation of sulfate aerosol scattering," J. Met. Soc. Japan, 77[6], 1299-1307 (1999).

6) A. C. Hirst, S. P. O'Farrell, H. B. Gordon, "Comparison of a coupled ocean-atmosphere model with and without oceanic eddy-induced advection. 1. Ocean spin-up and control integrations," J. Climate, 13[1], 139-163 (2000).

7) S. Valcke, R. Redler, OASIS4 User Guide (OASIS4 0 2), PRISM Report No 3, 2nd Ed. (2006), [PRISM web site, http://prism.enes.org].

8) C. Hill, C. DeLuca, V. Balaji, M. Suarez, A. da Silva, "The Architecture of the Earth System Modeling Framework," Comput. Sci. Eng., 6[1], 18-28 (2004).

9) J. Larson, R. Jacob, E. Ong, The Model Coupling Toolkit: A New Fortran90 Toolkit for Building Multiphysics Parallel Coupled Models, ANL/MCS-P1208-1204, Argonne National Laboratory (ANL) (2004).

10) W. D. Collins, C. M. Bitz, M. L. Blackmon, G. B. Bonan, C. S. Bretherton, J. A. Carton, P. Chang, S. C. Doney, J. J. Hack, T. B. Henderson, J. T. Kiehl, W. G. Large, D. S. McKenna, B. D. Santer, R. D. Smith, "The Community Climate System Model Version 3 (CCSM3)," J. Climate, 19[11], 2122-2143 (2006).

11) A. Craig, R. Jacob, B. Kauffman, T. Bettge, J. Larson, E. Ong, C. Ding, H. He, "CPL6: The new extensible, high-performance parallel coupler for the Community Climate System Model," Int. J. High Perf. Comp. App., 19[3], 309-328 (2005).

12) H. Nagai, T. Kobayashi, K. Tsuduki, K. Kim, Model Coupler for Coupling of Atmospheric, Oceanic, and Terrestrial Models, JAEA-Data/Code 2007-002, Japan Atomic Energy Agency (JAEA) (2007), [in Japanese].

13) H. Nagai, M. Chino, H. Terada, T. Harayama, T. Kobayashi, K. Tsuduki, K. Kim, A. Furuno, Numerical simulation system for environmental studies: SPEEDI-MP, JAEA-Research 2006-057, Japan Atomic Energy Agency (JAEA) (2006), [in Japanese].

14) H. Nagai, H. Terada, "Atmospheric models in the numerical simulation system (SPEEDI-MP) for environmental studies," Proc, Int. Symp. on Environmental Modeling and Radioecology, Rokkasho, Japan, Oct. 18-20, 2006, E126-E133 (2006).

15) N. Perlin, E. Skyllingstad, R. Samelson, P. Barbour, "Numerical simulation of air-sea coupling during coastal upwelling," $J$.
Phys. Oceanogr., 37[8], 2081-2093 (2007).

16) G. A. Grell, J. Dudhia, D. R. Stauffer, A description of the fifth-generation Penn State/NCAR Mesoscale Model (MM5), NCAR Tech. Note, NCAR/TN-398+STR, National Center for Atmospheric Research (1994).

17) W. C. Skamarock, J. B. Klemp, J. Dudhia, D. O. Gill, D. M. Barker, M. G. Duda, X. Huang, W. Wang, J. G. Powers, $A$ Description of the Advanced Research WRF Version 3, NCAR Tech. Note, NCAR/TN-475+STR, National Center for Atmospheric Research (2008).

18) H. Nagai, Atmosphere-soil-vegetation model including $\mathrm{CO}_{2}$ exchange processes: SOLVEG2, JAERI-Data/Code 2004-014, Japan Atomic Energy Research Institute (JAERI) (2004).

19) H. Nagai, "Incorporation of $\mathrm{CO}_{2}$ exchange processes into a multilayer atmosphere-soil-vegetation model," J. Appl. Meteor., 44[10], 1574-1592 (2005).

20) K. Tsuduki, T. Matsunaga, "Importance of hydrological parameters in contaminant transport modeling in a terrestrial environment," Proc, Int. Symp. on Environmental Modeling and Radioecology, Rokkasho, Japan, Oct. 18-20, 2006, E65-E72 (2006).

21) H. L. Tolman, User manual and system documentation of WAVWWATCH-III version 2.22, Tech. Note 222, NOAA/NWS/NCEP/OMB (2002).

22) G. L. Mellor, Users guide for a three-dimensional, primitive equation, numerical ocean model, Princeton University (1998).

23) K. Kim, H. Nagai, T. Yamashita, "Storm Surge Simulation on Hurricane Katrina in Gulf of Mexico Using Air-wave-sea Coupling Model," Full paper proc. of 4th Int. Conf. on Asian and Pacific Coasts APAC2007, Nanjing, China (2007).

24) H. Terada, M. Chino, "Development of an Atmospheric Dispersion Model for Accidental Discharge of Radionuclides with the Function of Simultaneous Prediction for Multiple Domains and its Evaluation by Application to the Chernobyl Nuclear Accident," J. Nucl. Sci. Technol., 45[9], 920-931 (2008).

25) T. Kobayashi, S. Otosaka, O. Togawa, K. Hayashi, "Development of a non-conservative radionuclides dispersion model in the ocean and its application to surface cesium-137 dispersion in the Irish Sea," J. Nucl. Sci. Technol., 44[2], 238-247 (2007).

26) T. Imamura, H. Koide, H. Takemiya, Stampi: A Message passing library for distributed parallel computing; User's guide, second edition, JAERI-Data/Code 2000-002, Japan Atomic Energy Research Institute (JAERI) (2000), [In Japanese].

27) H. Nagai, New Method for Model Coupling Using Stampi: Application to the Coupling of Atmosphere Model (MM5) and Land-surface Model (SOLVEG), JAERI-Data/Code 2003-021, Japan Atomic Energy Research Institute (JAERI) (2003).

28) G. L. Mellor, T. Yamada, "Development of a turbulence closure model for geophysical fluid problems," Rev. Geophys. Space Phys., 20[4], 851-875 (1982).

29) H. Terada, H. Nagai, A. Furuno, T. Kakefuda, T. Harayama, M. Chino, "Development of worldwide version of system for prediction of environmental emergency dose information; WSPEEDI 2nd version," Nihon-Genshiryoku-Gakkai Shi (J. At. Energy Soc. Jpn.), 7[3], 257-267 (2008), [in Japanese]. 